\title{
Temporal Lobe and Parietal Lobe Ependymal Tumor
}

National Cancer Institute

\section{Source}

National Cancer Institute. Temporal Lobe and Parietal Lobe Ependymal Tumor. NCI

Thesaurus. Code C131582.

An ependymal tumor affecting the temporal and parietal lobes of the brain. 